OPEN ACCESS

Edited by:

Martin Walter,

University of Tübingen, Germany

Reviewed by:

Gianfranco Spalletta,

Santa Lucia Foundation (IRCCS), Italy

Gabriele Ende,

University of Heidelberg, Germany

*Correspondence:

Marie Spies

marie.spies@meduniwien.ac.at

${ }^{\dagger}$ These authors have contributed equally to this work

Specialty section:

This article was submitted to Mood and Anxiety Disorders,

a section of the journal

Frontiers in Psychiatry

Received: 07 April 2020

Accepted: 18 August 2020

Published: 08 September 2020

Citation:

Silberbauer $L R$, Spurny $B$, Handschuh P, Klöbl M, Bednarik $P$, Reiter B, Ritter $V$, Trost $P$, Konadu ME,

Windpassinger M, Stimpfl T,

Bogner W, Lanzenberger $R$ and Spies $M$ (2020) Effect of Ketamine on

Limbic GABA and Glutamate: A

Human In Vivo Multivoxel Magnetic Resonance Spectroscopy Study.

Front. Psychiatry 11:549903. doi: 10.3389/fpsyt.2020.549903

\section{Effect of Ketamine on Limbic GABA and Glutamate: A Human In Vivo Multivoxel Magnetic Resonance Spectroscopy Study}

Leo R. Silberbauer ${ }^{1 \dagger}$, Benjamin Spurny ${ }^{1 \dagger}$, Patricia Handschuh ${ }^{1}$, Manfred Klöbl ${ }^{1}$, Petr Bednarik ${ }^{2}$, Birgit Reiter ${ }^{3}$, Vera Ritter ${ }^{1}$, Patricia Trost ${ }^{1}$, Melisande E. Konadu ${ }^{1}$, Marita Windpassinger ${ }^{4}$, Thomas Stimpfl ${ }^{3}$, Wolfgang Bogner ${ }^{2}$, Rupert Lanzenberger ${ }^{1}$ and Marie Spies ${ }^{1 \star}$

${ }^{1}$ Department of Psychiatry and Psychotherapy, Medical University of Vienna, Vienna, Austria, ${ }^{2}$ Department of Biomedical Imaging and Image-guided Therapy, High Field MR Centre, Medical University of Vienna, Vienna, Austria, ${ }^{3}$ Department of Laboratory Medicine, Medical University of Vienna, Vienna, Austria, ${ }^{4}$ Department of Anesthesia, Intensive Care Medicine and Pain Medicine, Medical University of Vienna, Vienna, Austria

Introduction: Converging evidence suggests that ketamine elicits antidepressant effects via enhanced neuroplasticity precipitated by a surge of glutamate and modulation of GABA. Magnetic resonance spectroscopic imaging (MRSI) illustrates changes to cerebral glutamate and GABA immediately following ketamine administration during dissociation. However, few studies assess subacute changes in the first hours following application, when ketamine's antidepressant effects emerge. Moreover, ketamine metabolites implicated in its antidepressant effects develop during this timeframe. Thus, this study aimed to investigate subacute changes in cerebral Glx (glutamate + glutamine), GABA and their ratio in seven brain regions central to depressive pathophysiology and treatment.

Methods: Twenty-five healthy subjects underwent two multivoxel MRS scans using a spiral encoded, MEGA-edited LASER-localized 3D-MRSI sequence, at baseline and $2 \mathrm{~h}$ following intravenous administration of racemic ketamine $(0.8 \mathrm{mg} / \mathrm{kg}$ bodyweight over $50 \mathrm{~min})$. Ketamine, norketamine and dehydronorketamine plasma levels were determined at routine intervals during and after infusion. Automated region-of-interest (ROI)-based quantification of mean metabolite concentration was used to assess changes in GABA+/ total creatine (tCr), Glx/tCr, and GABA+/Glx ratios in the thalamus, hippocampus, insula, putamen, rostral anterior cingulate cortex (ACC), caudal ACC, and posterior cingulate cortex. Effects of ketamine on neurotransmitter levels and association with ketamine- and metabolite plasma levels were tested with repeated measures analyses of variance (rmANOVA) and correlation analyses, respectively.

Results: For GABA+/tCr rmANOVA revealed a measurement by region interaction effect

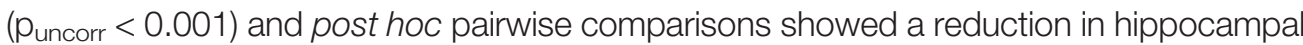
$\mathrm{GABA}+\mathrm{tCr}$ after ketamine ( $\left.\mathrm{p}_{\text {corr }}=0.02\right)$. For $\mathrm{Glx} / \mathrm{tCr}$ and $\mathrm{GABA}+/ \mathrm{Gl}$ neither main effects of measurement nor measurement by region interactions were observed (all puncorr $>$ 
0.05). Furthermore, no statistically significant associations between changes in any of the neurotransmitter ratios and plasma levels of ketamine, norketamine, or dehydronorketamine were observed ( $\left.p_{\text {corr }}>0.05\right)$.

Conclusion: This study provides evidence for decreased hippocampal GABA+/tCr ratio $2 \mathrm{~h}$ following ketamine administration. As MRS methodology measures total levels of intraand extracellular GABA, results might indicate drug induced alterations in GABA turnover. Our study in healthy humans suggests that changes in GABA levels, particularly in the hippocampus, should be further assessed for their relevance to ketamine's antidepressant effects.

Keywords: ketamine, GABA, glutamate, magnetic resonance spectroscopy, depression, ketamine metabolites, limbic system

\section{INTRODUCTION}

As a rapid acting and robust glutamatergic agent, ketamine occupies a unique position in antidepressant therapy. Numerous randomized controlled trials substantiate its efficacy in uni- and bipolar depression (1-3). The recent approval of an intranasal formulation by the U.S. Food and Drug Administration (FDA) and the European Medicines Agency (EMA) is likely to promote the use of ketamine in clinical care.

The glutamate model of depression implicates alterations to glutamate- and $\gamma$-aminobutyric acid (GABA)-related synaptic function (4); stress-related dysfunctional glutamate cycling is thought to result in excitotoxicity and neuronal atrophy. N-methylD-aspartate (NMDA)-receptor-mediated inhibition of GABA interneurons, resulting in reduced inhibitory control over prefrontal glutamate neurons and subsequent glutamate burst, are thought to facilitate ketamine's antidepressant effects (5). Accompanying $\alpha$-amino-3-hydroxy-5-methyl-4-isoxazolepropionic acid (AMPA)-receptor agonism activates second messenger pathways implicated in neuroplasticity (6). Thus, assessing brain glutamate and GABA levels provides insight into ketamine's antidepressant mechanisms of action.

Proton magnetic resonance spectroscopy ( $\left.{ }^{1} \mathrm{H}-\mathrm{MRS}\right)$ enables measurement of total tissue concentrations of glutamate (Glu), glutamine (Gln), pooled Glu and Gln (Glx) and GABA, among other metabolites in the living human brain. Gln is both glutamate precursor and metabolite; after Glu release, glia convert Glu to Gln, which is cycled back to the neuron (7). Consequently, Glx might be understood as an index of total glutamatergic potential (8).

MRS performed during or immediately after ketamine infusion shows increases in prefrontal cortex (PFC) glutamate (9-13), which is likely responsible for dissociative symptoms (14). Studies on the acute (up to 30 min postinfusion) influence of ketamine on GABA levels are currently limited. Two studies demonstrated increased PFC GABA levels $(11,15)$, though no change was observed in the thalamus (10).

Antidepressant effects emerge $2 \mathrm{~h}$ after infusion when acute effects, such as dissociation, have worn off and peak after $24 \mathrm{~h}(16$, 17). Estimation of Glx and GABA within a timeframe when antidepressant effects become evident is essential to understanding their relevance in ketamine's antidepressant properties. Previous studies on subacute changes show increase in pregenual anterior cingulate cortex (pgACC) $\mathrm{Gln} / \mathrm{Glu}$ ratio after $24 \mathrm{~h}$, which was not yet detected $1 \mathrm{~h}$ postinfusion $(18,19)$. Two other studies did not observe changes in Glu, Gln, Gln/Glu (20), nor GABA at various time points up to $48 \mathrm{~h}$ postinfusion (21). Interestingly, ketamine metabolites norketamine (norket) and dehydronorketamine (dhnk), to which antidepressant effects have been attributed in preclinical trials $(22,23)$, begin to develop during this time period (24). However, little is known about their glutamatergic and GABAergic effects.

Thus, research on ketamine's glutamate and GABA effects during this postdissociative timeframe, which is characterized by clinical antidepressant improvement, are currently limited. Furthermore, existing studies are restricted to single-voxel MRS approaches that do not allow for concomitant assessment of glutamate and GABA in multiple brain regions. However, we recently illustrated feasibility of a novel multivoxel 3D-magentic resonance spectroscopy imaging (MRSI) sequence with MEGALASER editing for parallel assessment of multiple regions of interest (25-27). In addition to propagating a topographically more extensive assessment of ketamine's effects, this multivoxel MRSI sequence allows for concomitant assessment of Glx and GABA+ (a combination of GABA and macromolecules), and thus also of their relation. Dysfunctionality of this relation has been discussed in the context of depressive pathophysiology $(5,8)$.

Here, we leverage 3D multivoxel MRSI technology to investigate ketamine's effects on Glx, GABA+, and their ratio in limbic brain regions implicated in depressive pathophysiology at a time when antidepressant effects of ketamine emerge. Regionally specific assessment during this time provides insight into the chronology of ketamine's neurotransmitter effects and their relevance for ketamine's antidepressant properties.

\section{METHODS}

\section{Subjects}

Twenty-five healthy male subjects (mean age $\pm \mathrm{SD}=26.76 \pm$ 4.71) were included in these analyses. Volunteers were recruited 
via postings on dedicated message boards at the Medical University of Vienna. Participants were free from internal, neurological or psychiatric disorders assessed via a thorough medical history, physical examination, electrocardiogram and routine laboratory parameters. The Structured Clinical Interview for DSM-IV Axis-I Disorders (SCID I) was administered by a psychiatrist in order to exclude any previous or current psychiatric diagnoses. Individuals had no history of substance use disorder and urine drug tests were performed at screening and each magnetic resonance imaging (MRI) session to exclude current drug use. Subjects were excluded at screening if they had any MRI contraindications. All participants provided written informed consent and received financial reimbursement for their participation. This study was approved by the Ethics Committee of the Medical University of Vienna and carried out according to the Declaration of Helsinki.

\section{Study Design and Ketamine Administration} Subjects underwent two MRI measurements. While the first measurement (MRI1) was performed without pharmacological challenge and served as a baseline scan, participants received 0.8 $\mathrm{mg} / \mathrm{kg}$ bodyweight racemic ketamine (Ketamine hydrochloride, $50 \mathrm{mg} / \mathrm{ml}$ ampoules, Hameln Pharma Plus $\mathrm{GmbH}$ ) intravenously over the course of $50 \mathrm{~min}$ starting $120 \mathrm{~min}$ prior to the second scan (MRI2) (see Figure S1). The dose of $0.8 \mathrm{mg} / \mathrm{kg}$ was chosen as it is within the subanesthetic, antidepressant dose range (1,2, 28). Vital parameters were monitored regularly and a clinician was present at all times.

\section{Magnetic Resonance Imaging}

MRI measurements were performed using a 64-channel head coil on a 3 Tesla MR Scanner (MAGNETOM Prisma, Siemens Medical, Erlangen, Germany) installed at the High-field MR
Center, Department of Biomedical Imaging and Image-guided Therapy, Medical University of Vienna. Structural T1-weighted images were acquired during each measurement using a standard magnetization-prepared rapid gradient-echo (MPRAGE) sequence $(\mathrm{TE}=1800 \mathrm{~ms}, \mathrm{TR}=2.37 \mathrm{~ms}, 208$ slices, $288 \times 288$ matrix size, slice thickness $0.85 \mathrm{~mm}$, voxel size $1.15 \times 1.15 \times$ $0.85 \mathrm{~mm}$ ) for accurate placement of the volume of interest (VOI) and mask extraction for automated region of interest (ROI)based analysis.

For spectroscopic measurements, a constant-density, spiralencoded, 3D-MRSI sequence with MEGA-LASER editing, as described in (25), was used. Real-time correction for rigid-body motion bias (i.e., translations and rotations) and correction of center frequency changes was applied $(25,29)$. Two MRSI measurements (MRSI1 and MRSI2) were performed consecutively to cover all ROIs and avoid inclusion of lipid-rich regions (see Figure 1). All MRS slices were placed parallel to the anterior commissure-posterior commissure line. Position of the VOIs of the second scanning session were determined based on VOI placement of the baseline measurement. VOI1 was centered to the medial part of the corpus callosum to cover the hippocampus and insula bilaterally, with a VOI $=80(\mathrm{l}-\mathrm{r}) \times 90(\mathrm{a}-\mathrm{p}) \times 80(\mathrm{~s}-\mathrm{i})$ $\mathrm{mm}^{3}$ and a field of view $(\mathrm{FOV})=160 \times 160 \times 160 \mathrm{~mm}^{3}$, see Figure 1. The acquired matrix size of $10 \times 10 \times 10$ (i.e., $\sim 4 \mathrm{~cm}^{3}$ nominal voxel size) was interpolated to a $16 \times 16 \times 16$ matrix (i.e., $\sim 1 \mathrm{~cm}^{3}$ nominal voxel size) during spectral processing steps. VOI2 was centered to cover the cingulate cortex with VOI $=80(\mathrm{l}-\mathrm{r}) \times 120(\mathrm{a}$ p) $\times 50(\mathrm{~s}-\mathrm{i}) \mathrm{mm}^{3}$ and field of view $($ FOV $)=160 \times 160 \times 160 \mathrm{~mm}^{3}$, see Figure 1. Siemens advanced shimming procedure with manual adjustments was used. During the EDIT-ON acquisition, MEGAediting pulses ( $60 \mathrm{~Hz}$ Gaussian pulses of $14.8 \mathrm{~ms}$ duration) were set to $1.9 \mathrm{ppm}$, editing the coupled $4 \mathrm{CH}_{2}$ triplet of GABA resonating at $3.02 \mathrm{ppm}(30,31)$. VOI selection via LASER and low-power and

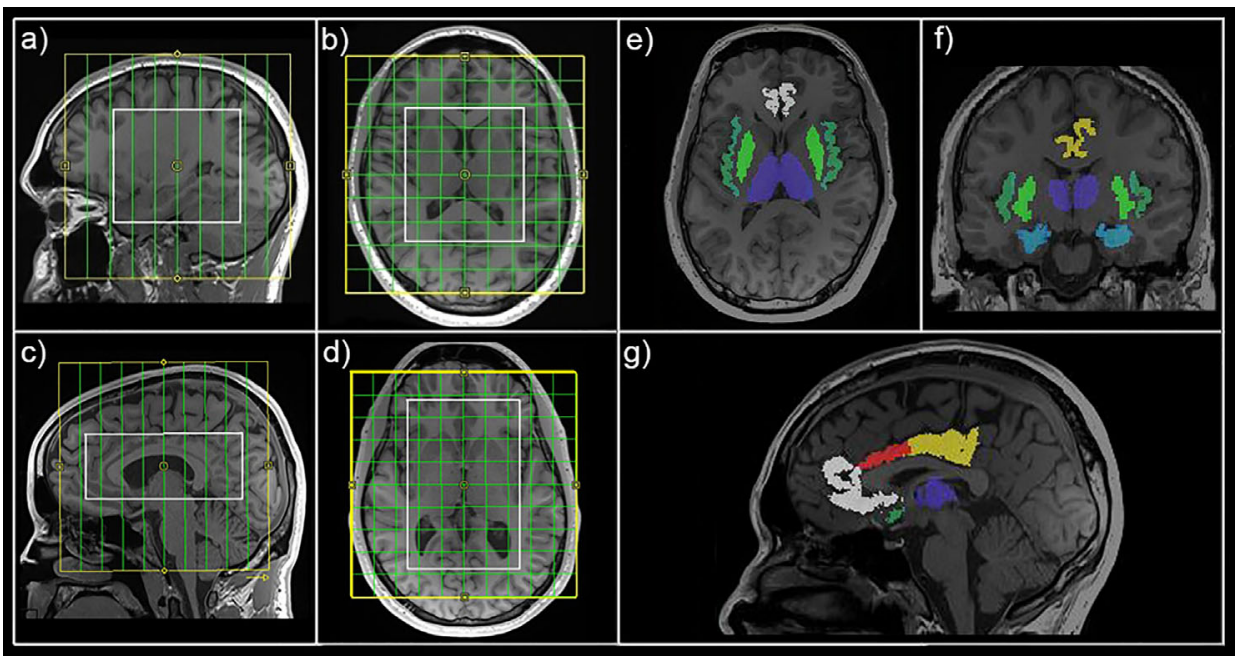

FIGURE 1 | Magnetic resonance spectroscopic imaging (MRSI) position and mask extraction. Field of view (yellow) and volume of interest (white) are shown for MRSI1 (A, B) and MRSI2 (C, D) in sagittal and horizontal view, respectively. Exemplary automated mask extraction with FreeSurfer is shown in horizontal (E), coronal (F), and sagittal (G) view showing masks for the thalamus (purple), hippocampus (light blue), insula (dark green), putamen (light green), rostral anterior cingulate cortex (white), caudal anterior cingulate cortex (red), and posterior cingulate cortex (yellow). 
wide-bandwidth GOIA pulses enabled MEGA editing with an echo time of $68 \mathrm{~ms}$ (25). For real-time correction, volumetric, dualcontrast, echo planar imaging-based navigators that update center frequency and head-position changes for each pair of EDIT-ON/ OFF acquisitions were used (i.e., with a repetition time of $1.6 \mathrm{~s}$, updated every $3.2 \mathrm{~s}$ ). For 3D-MRSI, 32 acquisition-weighted averages and two-step phase cycling were employed, resulting in a total scan time of 15:09 min per MRSI scan.

\section{Magnetic Resonance Spectroscopy Data Analyses}

All spectra within the VOI were processed automatically with an in-house software tool using MATLAB (R2013a, MathWorks, Natick, MA, USA), Bash (4.2.25, Free Software Foundation, Boston, MA, USA), MINC (2.0, MINC Tools, McConnell Brain Imaging Center, Montreal, QC, Canada) and LCModel software (6.3-1, S. Provencher, LCModel, Oakville, ON, Canada). Two different simulated basis sets were created using the GAMMA library, one for the non-edited spectra [containing 21 brain metabolites, including total creatine (tCr)] and one for the difference spectrum (containing GABA+ and Glx among others) $(32,33)$ (see Figure S2 for exemplary spectra). CramérRao lower bounds (CRLB) thresholds were set at 30\%. GABA+ and Glx ratios were calculated relatively to $\mathrm{tCr}(\mathrm{GABA}+/ \mathrm{tCr}$ and $\mathrm{Glx} / \mathrm{tCr}$ ). Automated ROI-based analyses were performed as previously described (26). FreeSurfer (6.0, https://surfer.nmr. mgh.harvard.edu/) $(34,35)$ was used for automated segmentation of structural T1-weighted images (see Figure 1). In-house MATLAB code was used to extract the ROIs (thalamus, hippocampus, insula, putamen, rostral anterior cingulate cortex (rACC), caudal anterior cingulate cortex (cACC), posterior cingulate cortex (PCC)) from each measurement. ROIs contained at least 2 voxels from the original $16 \times 16 \times 16$ grid (see Table S1 for volumetric properties of derived masks). GABA + and Glx (derived from the non-edited spectra) and tCr maps were interpolated to the resolution of structural images, overlaid with masks and mean $\mathrm{GABA}+/ \mathrm{tCr}, \mathrm{Glx} / \mathrm{tCr}$ and GABA+/Glx ratios calculated for each ROI. Metabolic data for thalamus, hippocampus, insula and putamen were derived from MRSI1 and rACC, CACC, and PCC from MRSI2. ROIs with $<90 \%$ valid interpolated voxels were excluded from analyses for the particular subject. ROIs were chosen based on their relevance to depressive pathophysiology, especially in the context of the glutamatergic depression model (5). rACC and cACC were investigated separately based on previous reports of ACC subregion-specific effects $(18,36)$.

\section{Ketamine and Metabolite Plasma Levels}

Venous blood samples were drawn at baseline and 20, 40, 50, 55, $60,70,80 \mathrm{~min}$ after ketamine administration, as well as immediately prior to and after MRI2. After centrifugation and separation of plasma, samples were frozen at $-80^{\circ} \mathrm{C}$ until analysis. Determination of ketamine, norket and dhnk plasma levels was accomplished using gas chromatography-mass spectrometry (GC-MS/MS) at the Clinical Department of
Laboratory Medicine, Medical University of Vienna, Austria. The applied method was validated according to the European Medicines Agency (EMA) guideline on bioanalytical method validation (37).

\section{Statistical Analyses}

SPSS Statistics (v26.0, 2010, SPSS, Inc., an IBM Company, Chicago, United States of America) and MATLAB (R2011a, MathWorks, Natick, MA, USA) were used for statistical analyses. Repeated measures analyses of variance (rmANOVA) with metabolite $(\mathrm{GABA}+/ \mathrm{tCr}, \mathrm{Glx} / \mathrm{tCr}$ or $\mathrm{GABA}+/ \mathrm{Glx})$ as dependent variable and measurement (MRI1, MRI2) and ROI (thalamus, hippocampus, insula, putamen, rACC, cACC, PCC) as within-subject factors were performed to probe for effects of ketamine on metabolite ratios. Interaction (measurement by region) and main effects (measurement, region) were tested.

Missing ratio values that failed to pass quality criteria (CRLB thresholds) were estimated using multiple imputation using ten repetitions and mean imputed values were used in subsequent analyses. See Table 1 for extent of imputed values per ROI and metabolite ratio. rACC was excluded from $\mathrm{GABA}+/ \mathrm{tCr}$ and GABA+/Glx models because missing values exceeded $40 \%$. Each metabolite ratio was estimated in an individual model. In case of significant main effect of measurement or interaction effects, post hoc pair-wise comparisons (t-test) were performed to isolate ROI-specific measurement effects. For Glx/tCr ratio, residuals were not consistently normally distributed (based on visual inspection of histograms). Logarithmic transformation of $\mathrm{Glx} / \mathrm{tCr}$ ratios was performed and rmANOVA was repeated, after which residuals showed normal distribution. Bonferroni procedure was used to correct for multiple comparisons. Associations between changes in neurotransmitter ratio from MRI1 to MRI2 (dependent variable) and ketamine, norket, and dhnk plasma levels (independent variables) were probed with spearman correlation analyses, as dependent variables did not show normal distribution (based on visual inspection of histograms). Plasma levels were interpolated to the time point of the start of each MRSI measurement for each subject using linear interpolation in MATLAB. Correlation analyses were performed for each ROI, neurotransmitter ratio and plasma level time-course. Again, Bonferroni procedure was utilized to correct for multiple comparisons. Ketamine, norket, and dhnk samples were available in 20 subjects. Raw MRSI data, without imputed values (see Table 1) was used to calculate MRI1 to MRI2 difference. As a result, sample sizes of correlation analysis were limited and we only report on ROIs with $\geq 10$ available data points. This was not the case for GABA+/tCr and GABA+/Glx ratios in the hippocampus, insula and rACC.

\section{RESULTS}

Mean \pm SD time between MRI1 and MRI2 was $67.84 \pm 72.43$ days. Mean \pm SD time between start of ketamine administration and MRSI data acquisition was $137.44 \pm 3.43 \mathrm{~min}$. See Table 2 
TABLE 1 | Number of imputed values, split by region of interest (ROI) and measurement.

\begin{tabular}{|c|c|c|c|c|c|}
\hline & $\mathrm{GABA}+/ \mathrm{tCr}$ & CRLB (\%) & $\mathrm{Glx} / \mathrm{tCr}$ & CRLB (\%) & GABA+/GIx \\
\hline Thalamus MRI1 & $1(4 \%)$ & 12.98 & $0(0 \%)$ & 8.60 & $1(4 \%)$ \\
\hline Thalamus MRI2 & $4(16 \%)$ & 12.91 & $3(12 \%)$ & 9.31 & $3(12 \%)$ \\
\hline Hippocampus MRI1 & $8(32 \%)$ & 17.18 & $1(4 \%)$ & 11.22 & $8(32 \%)$ \\
\hline Hippocampus MRI2 & $9(36 \%)$ & 17.66 & $3(12 \%)$ & 13.16 & $9(36 \%)$ \\
\hline Insula MRI1 & $5(20 \%)$ & 17.20 & $0(0 \%)$ & 10.69 & $5(20 \%)$ \\
\hline Insula MRI2 & $9(36 \%)$ & 16.38 & $3(12 \%)$ & 11.41 & $9(36 \%)$ \\
\hline Putamen MRI1 & $4(16 \%)$ & 13.22 & $0(0 \%)$ & 9.28 & $4(16 \%)$ \\
\hline Putamen MRI2 & $9(36 \%)$ & 14.38 & $3(12 \%)$ & 10.28 & $9(36 \%)$ \\
\hline rACC MRI1 & 17 (68\%) & $>30$ & $7(28 \%)$ & 13.44 & 17 (68\%) \\
\hline rACC MRI2 & 15 (60\%) & $>30$ & $4(16 \%)$ & 13.32 & 15 (60\%) \\
\hline cACC MRI1 & $9(36 \%)$ & 16.25 & $6(24 \%)$ & 9.85 & $9(36 \%)$ \\
\hline cACC MRI2 & $9(36 \%)$ & 15.65 & $3(12 \%)$ & 9.11 & $9(36 \%)$ \\
\hline PCC MRI1 & $9(36 \%)$ & 15.84 & $6(24 \%)$ & 9.31 & $9(36 \%)$ \\
\hline PCC MRI2 & $9(36 \%)$ & 15.94 & $3(12 \%)$ & 8.54 & $9(36 \%)$ \\
\hline
\end{tabular}

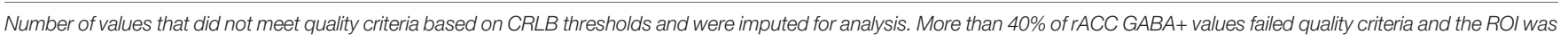

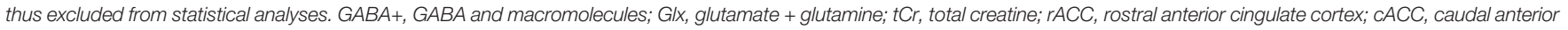
cingulate cortex; PCC, posterior cingulate cortex; CRLB, Cramér-Rao lower bounds.

for mean \pm SD GABA+/tCr, Glx/tCr, and GABA+/Glx for each ROI and measurement. Neurotransmitter ratios before and after ketamine application are displayed in Figure 2. See Table S2 and Figure 3 for average ketamine, norket, and dhnk plasma levels.

RmANOVA of GABA+/tCr showed a significant measurement by region interaction effect $\left(\mathrm{F}_{4.04,96.86}=6.17\right.$, puncorr $\left.<0.001\right)$, significant effect of region $\left(\mathrm{F}_{3.43,82.36}=34.27\right.$, $\left.\mathrm{p}_{\text {uncorr }}<0.001\right)$, but no significant effect of measurement $\left(\mathrm{F}_{1,24}=0.19\right.$, $\left.\mathrm{p}_{\text {uncorr }}=0.67\right)$. Post hoc pair-wise comparison revealed significantly higher $\mathrm{GABA}+/ \mathrm{tCr}$ at MRI1 than MRI2 in the hippocampus ( $\mathrm{p}_{\text {corr }}=0.02$ ), effects in insula $\left(\mathrm{p}_{\text {uncorr }}=0.03\right)$, putamen $\left(\mathrm{p}_{\text {uncorr }}=0.02\right)$, and PCC $\left(\mathrm{p}_{\text {uncorr }}=\right.$ 0.03 ), did not survive correction for multiple comparisons. For Glx/ $\mathrm{tCr}$ and $\mathrm{GABA}+/ \mathrm{Glx}$ neither measurement by region interaction effects $\left(\mathrm{Glx} / \mathrm{tCr}: \mathrm{F}_{3.46,82.92}=0.85, \mathrm{p}_{\text {uncorr }}=0.48\right.$; GABA+/Glx: $\mathrm{F}_{2.87,68.76}=2.34$, $\left.\mathrm{p}_{\text {uncorr }}=0.08\right)$, nor main effects of measurement $\left(\mathrm{Glx} / \mathrm{tCr}: \mathrm{F}_{1,24}=0.03, \mathrm{p}_{\text {uncorr }}=0.87 ; \mathrm{GABA}+/ \mathrm{Glx}: \mathrm{F}_{1,24}=1.05\right.$, puncorr $=0.32)$ were detected, though main effects of region were statistically significant $\left(\mathrm{Glx} / \mathrm{tCr}: \mathrm{F}_{3.37,80.83}=11.61\right.$, $\mathrm{p}_{\text {uncorr }}<0.001$; $\left.\mathrm{GABA}+/ \mathrm{Glx}: \mathrm{F}_{2.45,58.91}=44.79, \mathrm{p}_{\text {uncorr }}<0.001\right)$.
Based on Mauchly's test sphericity could not be assumed; Greenhouse-Geisser corrected results are given.

Before correction for multiple comparisons, correlation analyses revealed significant positive associations between GABA+/tCr difference MRI1 to MRI2 and ketamine plasma level $\left(\rho=0.66\right.$, $\left.p_{\text {uncorr }}=0.04\right)$ in the cACC, and with norket plasma level in the putamen $\left(\rho=0.84, \mathrm{p}_{\text {uncorr }}=0.002\right)$, as well as difference in Glx/tCr from MRI1 to MRI2 and dhnk plasma level in the hippocampus $\left(\rho=0.53, p_{\text {uncorr }}=0.03\right)$. However, none of these effects survived correction for multiple comparisons.

\section{DISCUSSION}

This multivoxel MRSI study assessed in vivo $\mathrm{Glx} / \mathrm{tCr}, \mathrm{GABA}+/ \mathrm{tCr}$, and GABA+/Glx $2 \mathrm{~h}$ following intravenous ketamine infusion in brain regions relevant to the glutamatergic theories of depression and antidepressant efficacy. We detected a significant reduction of $\mathrm{GABA}+/ \mathrm{tCr}$ ratio in the hippocampus. No statistically significant

TABLE 2 | Mean GABA+/tCr, Glx/tCr and GABA+/Glx ratios split by region of interest (ROI) and measurement.

\begin{tabular}{|c|c|c|c|c|c|c|c|c|}
\hline & & Thalamus & Hippocampus & Insula & Putamen & rACC & cACC & PCC \\
\hline & MRl1 & & & & & & & \\
\hline \multirow[t]{4}{*}{$\mathrm{GABA}+/ \mathrm{tCr}$} & $\begin{array}{c}\text { mean } \pm \text { SD } \\
\text { MRI2 }\end{array}$ & $0.31 \pm 0.07$ & $0.24 \pm 0.03$ & $0.25 \pm 0.04$ & $0.29 \pm 0.05$ & & $0.25 \pm 0.08$ & $0.25 \pm 0.06$ \\
\hline & mean \pm SD & $0.32 \pm 0.05$ & $0.22 \pm 0.04$ & $0.28 \pm 0.04$ & $0.31 \pm 0.03$ & & $0.24 \pm 0.07$ & $0.23 \pm 0.03$ \\
\hline & difference (\%) & 1.29 & -10.85 & 10.97 & 7.64 & & -3.68 & -7.91 \\
\hline & MRI1 & & & & & & & \\
\hline \multirow[t]{4}{*}{$\mathrm{G} \mid \mathrm{x} / \mathrm{tCr}$} & $\begin{array}{c}\text { mean } \pm \text { SD } \\
\mathrm{MRI} 2\end{array}$ & $1.53 \pm 0.47$ & $1.52 \pm 0.13$ & $1.59 \pm 0.16$ & $1.61 \pm 0.27$ & $1.83 \pm 0.68$ & $1.89 \pm 0.51$ & $1.70 \pm 0.29$ \\
\hline & mean \pm SD & $1.45 \pm 0.22$ & $1.45 \pm 0.26$ & $1.64 \pm 0.29$ & $1.61 \pm 0.29$ & $1.81 \pm 0.34$ & $1.94 \pm 0.67$ & $1.75 \pm 0.31$ \\
\hline & difference (\%) & -5.05 & -4.52 & 3.41 & 0.28 & -1.00 & 2.46 & 2.93 \\
\hline & MRI1 & & & & & & & \\
\hline \multirow[t]{3}{*}{$\mathrm{GABA}+/ \mathrm{Glx}$} & mean \pm SD & $0.22 \pm 0.05$ & $0.16 \pm 0.03$ & $0.16 \pm 0.02$ & $0.19 \pm 0.03$ & & $0.14 \pm 0.03$ & $0.15 \pm 0.03$ \\
\hline & mean + SD & $0.22+0.06$ & $0.14+0.03$ & $0.17+0.05$ & $0.19+0.06$ & & & $0.13+0.02$ \\
\hline & difference (\%) & 0.85 & -14.41 & 6.84 & 3.30 & & -1.87 & -8.81 \\
\hline
\end{tabular}

Raw data without imputed values is used; $r A C C$ GABA+/tCr and GABA+/Glx values did not meet quality criteria based on CRLB and were thus excluded from statistical analyses. GABA+, GABA and macromolecules; GIx, glutamate + glutamine; $t C r$, total creatine; rACC, rostral anterior cingulate cortex; $C A C C$, caudal anterior cingulate cortex; PCC, posterior cingulate cortex; CRLB, Cramér-Rao lower bounds. 

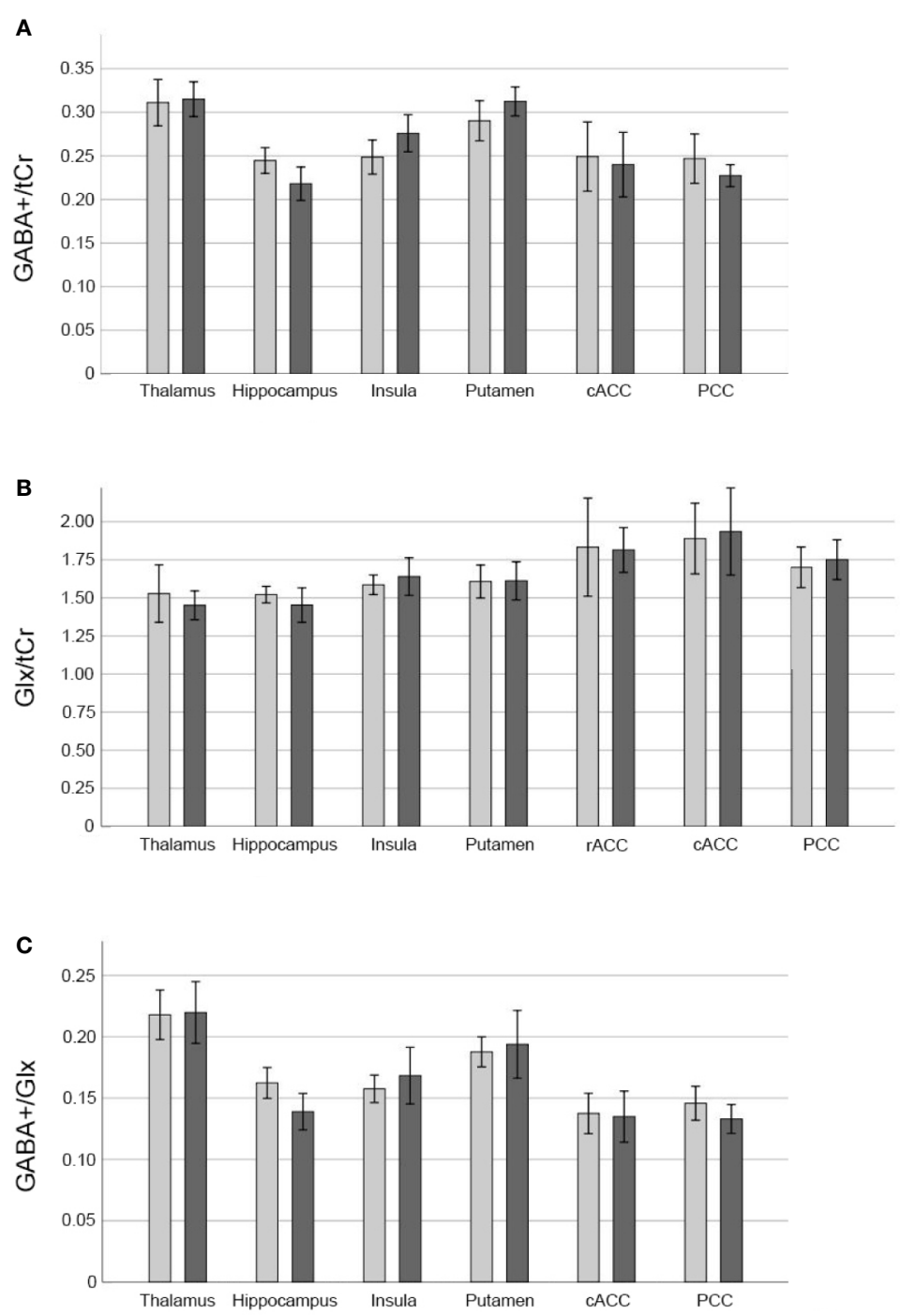

FIGURE 2 | Neurotransmitter ratios before and after ketamine. Bars denote mean neurotransmitter ratios [GABA+/tCr (A), GIx/tCr (B), GABA+/Glx (C)] before (light grey) and $2 \mathrm{~h}$ after infusion of $0.8 \mathrm{mg} / \mathrm{kg}$ bodyweight racemic ketamine (dark grey), brackets denote two standard errors. GABA+ values in the rACC did not meet quality criteria based on CRLB in more than $40 \%$ of cases and thus were excluded from statistical analyses. GABA+, GABA and macromolecules; Glx, glutamate + glutamine; tCr, total creatine; rACC, rostral anterior cingulate cortex; CACC, caudal anterior cingulate cortex; PCC, posterior cingulate cortex; CRLB, Cramér-Rao lower bounds.

changes in $\mathrm{Glx} / \mathrm{tCr}$ and GABA+/Glx ratios were observed. To the best of our knowledge, this is the first multivoxel MRS study to demonstrate subacute $(2 \mathrm{~h}$ ) effects of ketamine administration on limbic GABA levels in humans in vivo.

Timing MRS $2 \mathrm{~h}$ after ketamine administration assesses potential impact when dissociative effects wear off and ketamine's antidepressant properties unfold $(16,17)$. Thus, in theory, changes during this time period might be considered a temporally more related correlate of the substance's antidepressant effects. Despite this argument, previous human ketamine MRS studies on GABA levels were mostly limited to reports on immediate drug effects. While Stone et al. (10) did not observe acute changes in GABA following ketamine administration, two studies demonstrated increased GABA levels in the PFC during (11) and immediately after ketamine infusion (15). So far, the only study investigating subacute effects of ketamine on GABA concentration was performed by Valentine et al. (21). In this study, neither a change in occipital GABA, Glu nor Gln was observed 3 or $48 \mathrm{~h}$ after ketamine infusion in MDD. Our study differs from Valentine et al. (21) in that we assessed the hippocampus, among other regions, rather than the occipital cortex. These regional differences bring to light the importance of a regionally extensive investigation. Using multivoxel MRSI, seven ROIs were selected based on their relevance to depressive pathophysiology. Though assessment of several ROIs as we did also requires a-priori selection, bias is likely nevertheless lower than that resulting from single-voxel investigations which limit results and their interpretation to a single region. In addition, we assessed healthy controls rather than patients with MDD. 


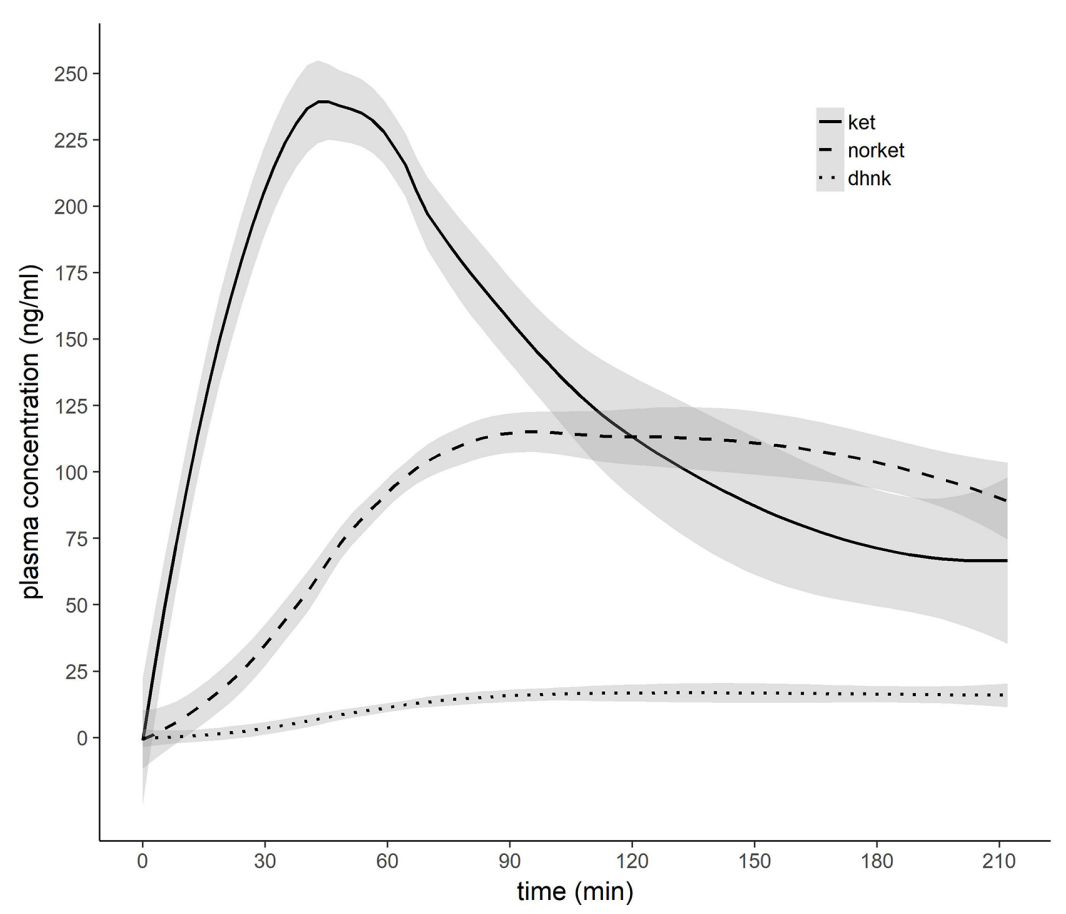

FIGURE 3 | Pharmacokinetic profile of ketamine, norketamine, and dehydronorketamine. $0.8 \mathrm{mg} / \mathrm{kg}$ bodyweight racemic ketamine were infused over 50 min. X-axis indicates minutes after initiation of ketamine infusion. Grey ribbons indicate standard error of the mean. Ket, ketamine; norket, norketamine;

dhnk, dehydronorketamine.

Though this limits the extent to which changes we observe can be interpreted as direct facilitators of antidepressant efficacy, our investigation highlights changes that should be assessed in future studies in MDD. In addition, our focus on ROIs that are central to depressive pathophysiology increases potential for detection of clinically relevant changes that can then be validated in future patient studies.

Our current data may at first appear inconsistent with previous reports of reduced GABA levels in depression (38) and normalization following antidepressant treatment such as with selective serotonin reuptake inhibitors and electroconvulsive therapy $(39,40)$. Given the sparse human data on subacute effects of ketamine on GABA, preclinical data may provide guidance on this point. Increases in GABA levels were reported $30 \mathrm{~min}$ but not $24 \mathrm{~h}$ after ketamine administration in prefrontal regions in rats (41). Perrine et al. (42) reported decreased GABA levels $24 \mathrm{~h}$ after ketamine administration in the ACC. Moreover, reduced GABA in parvalbumin (PV) interneurons of the PFC was demonstrated $2 \mathrm{~h}$ after ketamine administration by Zhou et al. (43). Thus, various preclinical studies suggest GABA reduction within the subacute timeframe (2-24 h). In accordance with our hippocampus findings, Wang et al. (44) reported immediate decreases in GABA levels in hippocampal PV interneurons in rats following ketamine. Decreases in GABA levels as reported by Wang et al. (44) and (43) were associated with downregulation of GAD67, a protein implicated in the glutamate/GABA-glutamine cycle, suggesting alterations in GABA turnover (45). Thus, though in contrast with some human in vivo reports, our results are in line with various preclinical studies suggesting subacute GABA reduction after ketamine.

We did not detect statistically significant effects on Glx/tCr. Preclinical studies report a rapid glutamate surge immediately following ketamine administration (5). Human MRS demonstrates concomitant changes in Glu, Gln and Glx levels (9-13). However, similar to GABA, previous studies on subacute effects of ketamine on Glu and Gln are also limited. Evans et al. (20) did not detect changes in Glu, Gln or Gln/Glu in the pgACC 24 h after ketamine administration in MDD. In contrast, $\mathrm{Li}$ et al. report increased ACC Gln/Glu $24 \mathrm{~h}$ after infusion in healthy individuals. This effect was not yet present at $1 \mathrm{~h}$ and was restricted to the pgACC $(18,19)$. Li et al. interpret this regional specificity to be reflective of AMPA distribution, more specifically AMPA/NMDA ratio, which is higher in the pgACC compared to anterior midcingulate cortex (aMCC) $(36,46)$. We did not detect regional differences within the ACC after $2 \mathrm{~h}$, though our ROIs (rACC, cACC) do not entirely overlap with pgACC and aMCC as delineated by $(18,19)$.

MRS ketamine literature exhibits heterogeneous outcome parameters; we assessed GABA+/tCr, Glx/tCr, and GABA+/Glx. With the exception of $\mathrm{Gln} / \mathrm{Glu}$, which is considered indicative of alterations in turnover and seen as an index of release (47), MRS directly assesses changes in total neurotransmitter levels; alterations to relative intra/extracellular concentrations, as would be postulated in release or cell-cell (neuron-glial) cycling, are not primarily detected. Any effects on $\mathrm{Glx} / \mathrm{tCr}$ or $\mathrm{GABA}+/ \mathrm{tCr}$ are reflective of change in total concentration, for example via alterations in turnover. The decreased GABA+/tCr ratio observed in this study 
might be interpreted within this context. Synthesis and degradation of GABA and glutamate within the glutamate/GABA-glutamine cycle are shown to be closely tied to the tricarboxylic acid (TCA) cycle, which is central to oxidative energy metabolism (7). Changes in GABA and glutamate concentrations likely occur dynamically and in an activity-dependent manner on a short timescale of min $(11,48)$. Thus, it is plausible that ketamine might induce changes in total concentration of Glx and GABA detectable with MRS within our measurement timeframe. Along this line, rodent studies suggest a modest acute increase in de novo glutamate $(\sim 18 \%)$ and GABA ( $10 \%)$ synthesis following ketamine administration in the medial PFC (mPFC). No changes, however, were observed in the hippocampus (49). Though our human in vivo MRS study detected decreases in hippocampal $\mathrm{GABA}+/ \mathrm{tCr}$ ratio, the aforementioned studies highlight that changes to GABA or glutamate turnover are feasible within our measurement timeframe. The only previous MRS studies showing subacute glutamatergic alterations after ketamine infusion assess Gln/Glu $(18,19)$. However, changes in Gln/Glu could coincide with constant Glx, in which case they would not be observed in our study.

We investigated possible changes in GABA+/Glx ratio based on previous evidence of imbalanced excitatory and inhibitory neurotransmission in depressive pathophysiology and in relation to antidepressant efficacy (50). Previous studies indicate that rapid acting antidepressants might restore deficits in this balance by enhancing GABAergic neurotransmission (51). While we find downregulation of hippocampal GABA levels within the subacute period, we did not detect a statistically significant effect of ketamine on GABA+/Glx ratios in any ROI. Thus, our results do not provide evidence that ketamine infusion affects balance of GABA and glutamate levels $2 \mathrm{~h}$ after administration. Given ketamine's synchronized glutamatergic and GABAergic effects (5), studies on GABA+/Glx at other time points should be assessed in future studies.

We specifically probed alterations to $\mathrm{GABA}+/ \mathrm{tCr}, \mathrm{Glx} / \mathrm{tCr}$, and GABA+/Glx because we postulated that assessment of changes during the period of clinical efficacy may provide insight into antidepressant mechanisms of action. While decreased hippocampal GABA/tCr sheds light on the importance of the GABAergic system in the antidepressant efficacy of ketamine, other therapeutic mechanisms of action have previously been shown to be relevant. Most often discussed among these processes is neuroplasticity activated by modulation of the glutamate and GABA systems. Ketamine administration increases spine formation by activation of the mTOR pathway in rodents (52), a process that is considered to reverse neuronal atrophy associated with depressive pathophysiology (53). Hippocampal volume has repeatedly been implicated in the pathophysiology of MDD (54) and changes in structure and function of the hippocampus are associated with depressive symptoms across psychiatric diagnoses, e.g. in schizophrenia (55), and are even present in subclinical depression (56). Moreover, hippocampal volume is associated with antidepressant response to traditional antidepressants (57) and ketamine (58). Preclinical data suggest that ketamine induces synaptic and vascular alterations of the hippocampus $24 \mathrm{~h}$ after administration (59). However, the impact of reduced $\mathrm{GABA}+/ \mathrm{tCr}$ ratio $2 \mathrm{~h}$ after administration on ketamineinduced hippocampal neuroplasticity remains to be elucidated.

We did not observe a statistically significant association between plasma levels of major ketamine metabolites (norket, dhnk) and changes in neurotransmitter ratios. This is in line with a previous investigation by Milak et al. (11) that did not observe a correlation between norket and dhnk levels and Glx and GABA levels during administration. While antidepressant-like effects of ketamine metabolites were demonstrated in preclinical trials, the role of glutamate and GABA in their effects is currently not clear (60). Rodent studies show promising clinical antidepressant effects for hydroxynorketamine (hnk). However, we did not assess this metabolite in our study because levels typically peak $24 \mathrm{~h}$ after ketamine application (61).

Strengths of this investigation include use of a novel multivoxel MRSI sequence that allows for the hitherto regionally most extensive simultaneous assessment of human in vivo glutamatergic and GABAergic effects after ketamine exposure. Assessment of effects when clinical antidepressant properties emerge allows for probing of the correlates of antidepressant improvement itself while complementing existing literature on more short- and long-term changes. We thus contribute to a chronological framework for understanding ketamine's glutamatergic and GABAergic effects.

Our study is not without limitations. This investigation does not follow a randomized, controlled study design. However, given ketamine's unambiguous dissociative effects, an adequate control condition is challenging (62) and application of GABAergic compounds such as benzodiazepines, as performed in previous trials (2) is particularly questionable when GABA levels are assessed. The sample size $(n=25)$ of our investigation should be considered a limitation, nevertheless it exceeds those of the majority of previous pharmacological MRS studies (9-12, 15, 20, $21,63)$ and is a result of our resource intensive methodology. In this context, the inclusion of imputed MRS data should also be discussed. We performed stringent quality control (CRLB thresholds) on MRS data and while this results in missing values, imputation allows for application of statistical procedures. However, it should be taken into consideration that mean imputation as performed reduces variance and may thus make subthreshold effects more pronounced. Extent of missing- and subsequently imputed values within an ROI may thus affect results. Moreover, the applied multivoxel MRSI sequence does not allow for discrimination of Glu and Gln, thus Gln/Glu is not assessed. Furthermore, signal spill-over from adjacent voxels cannot be excluded. Lastly, venous blood samples were not acquired during performance of MR measurements. This issue was, however, circumvented through interpolation of plasma curves.

In conclusion, we demonstrate a statistically significant decrease in GABA+/tCr in the hippocampus $2 \mathrm{~h}$ after ketamine infusion in healthy individuals. No statistically significant changes in Glx/tCr and GABA+/Glx were observed in any of the assessed ROIs. These results suggest changes to GABA turnover in the subacute time period after ketamine administration, in accordance with preclinical data $(43,44)$. Our findings, acquired during the time-period when antidepressant effects emerge, highlight a 
potential role for hippocampal GABAergic neurotransmission in ketamine's antidepressant effects, though validation in MDD is required. This study thus contributes to our understanding of the chronology of neurotransmitter changes after ketamine administration.

\section{DATA AVAILABILITY STATEMENT}

The datasets presented in this article are not readily available due to ethical reasons. Please contact marie.spies@meduniwien.ac.at for questions.

\section{ETHICS STATEMENT}

This study was reviewed and approved by the Ethics Committee of the Medical University of Vienna. The participants provided their written informed consent to participate in this study.

\section{AUTHOR CONTRIBUTIONS}

MS and RL designed the study. LS, BS, and MS wrote the manuscript. Data analyses were performed by BS under supervision of MS, MK, and WB and contribution of LS. LS, $\mathrm{PH}, \mathrm{PT}, \mathrm{MEK}, \mathrm{MW}$, and MS provided medical support. VR provided administrative support. BR and TS performed analyses of plasma levels of ketamine and its metabolites. BS performed MR measurements. MS was scientific supervisor of the study and principal investigator. All authors contributed to the article and approved the submitted version.

\section{REFERENCES}

1. Zarate CAJr., Brutsche NE, Ibrahim L, Franco-Chaves J, Diazgranados N, Cravchik A, et al. Replication of ketamine's antidepressant efficacy in bipolar depression: a randomized controlled add-on trial. Biol Psychiatry (2012) 71:939-46. doi: 10.1016/j.biopsych.2011.12.010

2. Fava M, Freeman MP, Flynn M, Judge H, Hoeppner BB, Cusin C, et al. Double-blind, placebo-controlled, dose-ranging trial of intravenous ketamine as adjunctive therapy in treatment-resistant depression (TRD). Mol Psychiatry (2020) 25:1592-603. doi: 10.1038/s41380-018-0256-5

3. Phillips JL, Norris S, Talbot J, Birmingham M, Hatchard T, Ortiz A, et al. Single, Repeated, and Maintenance Ketamine Infusions for TreatmentResistant Depression: A Randomized Controlled Trial. Am J Psychiatry (2019) 176:401-9. doi: 10.1176/appi.ajp.2018.18070834

4. Sanacora G, Treccani G, Popoli M. Towards a glutamate hypothesis of depression: an emerging frontier of neuropsychopharmacology for mood disorders. Neuropharmacology (2012) 62:63-77. doi: 10.1016/j.neuropharm. 2011.07.036

5. Abdallah CG, Sanacora G, Duman RS, Krystal JH. Ketamine and rapid-acting antidepressants: a window into a new neurobiology for mood disorder therapeutics. Annu Rev Med (2015b) 66:509-23. doi: 10.1146/annurev-med053013-062946

6. Duman RS, Aghajanian GK, Sanacora G, Krystal JH. Synaptic plasticity and depression: new insights from stress and rapid-acting antidepressants. Nat Med (2016) 22:238. doi: 10.1038/nm.4050

7. Bak LK, Schousboe A, Waagepetersen HS. The glutamate/GABA-glutamine cycle: aspects of transport, neurotransmitter homeostasis and ammonia transfer. J Neurochem (2006) 98:641-53. doi: 10.1111/j.1471-4159.2006.03913.x

\section{FUNDING}

This project was funded by Brain and Behavior Research Foundation (formerly NARSAD) Young Investigator grants to MS (23741) and PB (27238) as well as grants from the Austrian Science Fund (FWF, KLI516) and the Vienna Science and Technology Fund (WWTF, CS18-039) awarded to RL. Additional support was provided by the Medical University of Vienna's Medical Imaging Cluster and a grant from the Austrian Science Fund (FWF, P30701) awarded to WB. LS and MK are recipients of DOC fellowships of the Austrian Academy of Sciences at the Department of Psychiatry and Psychotherapy, Medical University of Vienna. PB is recipient of a Marie Skłodowska-Curie Individual Fellowship (846793). MEK is a Medical University of Vienna MD PhD Excellence Program awardee. Funding sources had no further role in study design, collection, analyses and interpretation of data, writing of the report; nor in the decision to submit the paper for publication.

\section{ACKNOWLEDGMENTS}

We thank PM, GM, JU, and JJ for medical support as well as ES for administrative support.

\section{SUPPLEMENTARY MATERIAL}

The Supplementary Material for this article can be found online at: https://www.frontiersin.org/articles/10.3389/fpsyt.2020. 549903/full\#supplementary-material

8. Lener MS, Niciu MJ, Ballard ED, Park M, Park LT, Nugent AC, et al. Glutamate and Gamma-Aminobutyric Acid Systems in the Pathophysiology of Major Depression and Antidepressant Response to Ketamine. Biol Psychiatry (2017) 81:886-97. doi: 10.1016/j.biopsych.2016.05.005

9. Rowland LM, Bustillo JR, Mullins PG, Jung RE, Lenroot R, Landgraf E, et al. Effects of ketamine on anterior cingulate glutamate metabolism in healthy humans: a 4-T proton MRS study. Am J Psychiatry (2005) 162:394-6. doi: 10.1176/appi.ajp.162.2.394

10. Stone JM, Dietrich C, Edden R, Mehta MA, De Simoni S, Reed LJ, et al. Ketamine effects on brain GABA and glutamate levels with $1 \mathrm{H}$-MRS: relationship to ketamine-induced psychopathology. Mol Psychiatry (2012) 17:664-5. doi: 10.1038/mp.2011.171

11. Milak MS, Proper CJ, Mulhern ST, Parter AL, Kegeles LS, Ogden RT, et al. A pilot in vivo proton magnetic resonance spectroscopy study of amino acid neurotransmitter response to ketamine treatment of major depressive disorder. Mol Psychiatry (2016) 21:320-7. doi: 10.1038/mp.2015.83

12. Kraguljac NV, Frolich MA, Tran S, White DM, Nichols N, Barton-Mcardle A, et al. Ketamine modulates hippocampal neurochemistry and functional connectivity: a combined magnetic resonance spectroscopy and resting-state fMRI study in healthy volunteers. Mol Psychiatry (2017) 22:562-9. doi: $10.1038 / \mathrm{mp} .2016 .122$

13. Javitt DC, Carter CS, Krystal JH, Kantrowitz JT, Girgis RR, Kegeles LS, et al. Utility of Imaging-Based Biomarkers for Glutamate-Targeted Drug Development in Psychotic Disorders: A Randomized Clinical Trial. JAMA Psychiatry (2018) 75:11-9. doi: 10.1001/jamapsychiatry.2017.3572

14. Anand A, Charney DS, Oren DA, Berman RM, Hu XS, Cappiello A, et al. Attenuation of the neuropsychiatric effects of ketamine with lamotrigine: support for hyperglutamatergic effects of $\mathrm{N}$-methyl-D-aspartate receptor 
antagonists. Arch Gen Psychiatry (2000) 57:270-6. doi: 10.1001/archpsyc. 57.3.270

15. Rodriguez CI, Kegeles LS, Levinson A, Ogden RT, Mao X, Milak MS, et al. In vivo effects of ketamine on glutamate-glutamine and gamma-aminobutyric acid in obsessive-compulsive disorder: Proof of concept. Psychiatry Res (2015) 233:141-7. doi: 10.1016/j.pscychresns.2015.06.001

16. Berman RM, Cappiello A, Anand A, Oren DA, Heninger GR, Charney DS, et al. Antidepressant effects of ketamine in depressed patients. Biol Psychiatry (2000) 47:351-4. doi: 10.1016/S0006-3223(99)00230-9

17. Zarate CAJr., Singh JB, Carlson PJ, Brutsche NE, Ameli R, Luckenbaugh DA, et al. A randomized trial of an N-methyl-D-aspartate antagonist in treatmentresistant major depression. Arch Gen Psychiatry (2006) 63:856-64. doi: 10.1001/archpsyc.63.8.856

18. Li M, Demenescu LR, Colic L, Metzger CD, Heinze HJ, Steiner J, et al. Temporal Dynamics of Antidepressant Ketamine Effects on Glutamine Cycling Follow Regional Fingerprints of AMPA and NMDA Receptor Densities. Neuropsychopharmacology (2017) 42:1201-9. doi: 10.1038/ npp.2016.184

19. Li M, Woelfer M, Colic L, Safron A, Chang C, Heinze HJ, et al. Default mode network connectivity change corresponds to ketamine's delayed glutamatergic effects. Eur Arch Psychiatry Clin Neurosci (2020) 270:207-16. doi: 10.1007/ s00406-018-0942-y

20. Evans JW, Lally N, An L, Li N, Nugent AC, Banerjee D, et al. 7T (1)HMRS in major depressive disorder: a Ketamine Treatment Study. Neuropsychopharmacology (2018) 43:1908-14. doi: 10.1038/s41386-018-0057-1

21. Valentine GW, Mason GF, Gomez R, Fasula M, Watzl J, Pittman B, et al. The antidepressant effect of ketamine is not associated with changes in occipital amino acid neurotransmitter content as measured by [(1)H]-MRS. Psychiatry Res (2011) 191:122-7. doi: 10.1016/j.pscychresns.2010.10.009

22. Salat K, Siwek A, Starowicz G, Librowski T, Nowak G, Drabik U, et al. Antidepressant-like effects of ketamine, norketamine and dehydronorketamine in forced swim test: Role of activity at NMDA receptor. Neuropharmacology (2015) 99:301-7. doi: 10.1016/j.neuropharm.2015.07.037

23. Yang C, Kobayashi S, Nakao K, Dong C, Han M, Qu Y, et al. AMPA Receptor Activation-Independent Antidepressant Actions of Ketamine Metabolite (S)Norketamine. Biol Psychiatry (2018) 84:591-600. doi: 10.1016/j.biopsych. 2018.05.007

24. Zhao X, Venkata SL, Moaddel R, Luckenbaugh DA, Brutsche NE, Ibrahim L, et al. Simultaneous population pharmacokinetic modelling of ketamine and three major metabolites in patients with treatment-resistant bipolar depression. Br J Clin Pharmacol (2012) 74:304-14. doi: 10.1111/j.13652125.2012.04198.x

25. Bogner W, Gagoski B, Hess AT, Bhat H, Tisdall MD, Van Der Kouwe AJ, et al. 3D GABA imaging with real-time motion correction, shim update and reacquisition of adiabatic spiral MRSI. Neuroimage (2014a) 103:290-302. doi: 10.1016/j.neuroimage.2014.09.032

26. Spurny B, Heckova E, Seiger R, Moser P, Klobl M, Vanicek T, et al. Automated ROI-Based Labeling for Multi-Voxel Magnetic Resonance Spectroscopy Data Using FreeSurfer. Front Mol Neurosci (2019) 12:28. doi: 10.3389/ fnmol.2019.00028

27. Spurny B, Seiger R, Moser P, Vanicek T, Reed MB, Heckova E, et al. Hippocampal GABA levels correlate with retrieval performance in an associative learning paradigm. Neuroimage (2020) 204:116244. doi: 10.1016/ j.neuroimage.2019.116244

28. Perry EBJr., Cramer JA, Cho HS, Petrakis IL, Karper LP, Genovese A, et al. Psychiatric safety of ketamine in psychopharmacology research. Psychopharmacol (Berl) (2007) 192:253-60. doi: 10.1007/s00213-007-0706-2

29. Bogner W, Hess AT, Gagoski B, Tisdall MD, Van Der Kouwe AJ, Trattnig S, et al. Real-time motion- and B0-correction for LASER-localized spiralaccelerated 3D-MRSI of the brain at 3T. Neuroimage (2014b) 88:22-31. doi: 10.1016/j.neuroimage.2013.09.034

30. Andronesi OC, Ramadan S, Ratai EM, Jennings D, Mountford CE, Sorensen AG. Spectroscopic imaging with improved gradient modulated constant adiabaticity pulses on high-field clinical scanners. J Magn Reson (2010) 203:283-93. doi: 10.1016/j.jmr.2010.01.010

31. Mullins PG, Mcgonigle DJ, O'gorman RL, Puts NA, Vidyasagar R, Evans CJ, et al. Current practice in the use of MEGA-PRESS spectroscopy for the detection of GABA. Neuroimage (2014) 86:43-52. doi: 10.1016/j.neuroimage. 2012.12.004

32. Smith SA, Levante TO, Meier BH, Ernst RR. Computer Simulations in Magnetic Resonance. An Object-Oriented Programming Approach. J Magnetic Resonance Ser A (1994) 106:75-105. doi: 10.1006/jmra.1994.1008

33. Hnilicova P, Povazan M, Strasser B, Andronesi OC, Gajdosik M, Dydak U, et al. Spatial variability and reproducibility of GABA-edited MEGA-LASER 3D-MRSI in the brain at 3 T. NMR BioMed (2016) 29:1656-65. doi: 10.1002/ nbm. 3613

34. Fischl B, Salat DH, Busa E, Albert M, Dieterich M, Haselgrove C, et al. Whole brain segmentation: automated labeling of neuroanatomical structures in the human brain. Neuron (2002) 33:341-55. doi: 10.1016/s0896-6273(02)00569-x

35. Desikan RS, Segonne F, Fischl B, Quinn BT, Dickerson BC, Blacker D, et al. An automated labeling system for subdividing the human cerebral cortex on MRI scans into gyral based regions of interest. Neuroimage (2006) 31:968-80. doi: 10.1016/j.neuroimage.2006.01.021

36. Palomero-Gallagher N, Vogt BA, Schleicher A, Mayberg HS, Zilles K. Receptor architecture of human cingulate cortex: evaluation of the fourregion neurobiological model. Hum Brain Mapp (2009) 30:2336-55. doi: $10.1002 / \mathrm{hbm} .20667$

37. European Medicines Agency. Guideline on bioanalytical method validation, EMEA/CHMP/EWP/192217/2009 Rev, Vol. 1. (2011), Corr 2**. Retrieved from https://www.ema.europa.eu/en/documents/scientific-guideline/ guideline-bioanalytical-method-validation_en.pdf (02.09.2020)

38. Schur RR, Draisma LW, Wijnen JP, Boks MP, Koevoets MG, Joels M, et al. Brain GABA levels across psychiatric disorders: A systematic literature review and meta-analysis of (1) H-MRS studies. Hum Brain Mapp (2016) 37:333752. doi: $10.1002 / \mathrm{hbm} .23244$

39. Sanacora G, Mason GF, Rothman DL, Krystal JH. Increased occipital cortex GABA concentrations in depressed patients after therapy with selective serotonin reuptake inhibitors. Am J Psychiatry (2002) 159:663-5. doi: 10.1176/appi.ajp.159.4.663

40. Sanacora G, Mason GF, Rothman DL, Hyder F, Ciarcia JJ, Ostroff RB, et al. Increased Cortical GABA Concentrations in Depressed Patients Receiving ECT. Am J Psychiatry (2003) 160:577-9. doi: 10.1176/appiajp.160.3.577

41. Chowdhury GMI, Zhang J, Thomas M, Banasr M, Ma X, Pittman B, et al. Transiently increased glutamate cycling in rat PFC is associated with rapid onset of antidepressant-like effects. Mol Psychiatry (2017) 22:120-6. doi: $10.1038 / \mathrm{mp} .2016 .34$

42. Perrine SA, Ghoddoussi F, Michaels MS, Sheikh IS, Mckelvey G, Galloway MP. Ketamine reverses stress-induced depression-like behavior and increased GABA levels in the anterior cingulate: an 11.7 T 1H-MRS study in rats. Prog Neuropsychopharmacol Biol Psychiatry (2014) 51:9-15. doi: 10.1016/ j.pnpbp.2013.11.003

43. Zhou Z, Zhang G, Li X, Liu X, Wang N, Qiu L, et al. Loss of Phenotype of Parvalbumin Interneurons in Rat Prefrontal Cortex Is Involved in Antidepressant- and Propsychotic-Like Behaviors Following Acute and Repeated Ketamine Administration. Mol Neurobiol (2015) 51:808-19. doi: 10.1007/s12035-014-8798-2

44. Wang N, Zhang G-F, Liu X-Y, Sun H-L, Wang X-M, Qiu L-L, et al. Downregulation of Neuregulin 1-ErbB4 Signaling in Parvalbumin Interneurons in the Rat Brain May Contribute to the Antidepressant Properties of Ketamine. J Mol Neurosci (2014) 54:211-8. doi: 10.1007/s12031-014-0277-8

45. Pham TH, Gardier AM. Fast-acting antidepressant activity of ketamine: highlights on brain serotonin, glutamate, and GABA neurotransmission in preclinical studies. Pharmacol Ther (2019) 199:58-90. doi: 10.1016/ j.pharmthera.2019.02.017

46. Maeng S, Zarate CAJr., Du J, Schloesser RJ, Mccammon J, Chen G, et al. Cellular mechanisms underlying the antidepressant effects of ketamine: role of alpha-amino-3-hydroxy-5-methylisoxazole-4-propionic acid receptors. Biol Psychiatry (2008) 63:349-52. doi: 10.1016/j.biopsych.2007.05.028

47. Iltis I, Koski DM, Eberly LE, Nelson CD, Deelchand DK, Valette J, et al. Neurochemical changes in the rat prefrontal cortex following acute phencyclidine treatment: an in vivo localized (1)H MRS study. NMR BioMed (2009) 22:737-44. doi: 10.1002/nbm.1385

48. Bednarik P, Tkac I, Giove F, Dinuzzo M, Deelchand DK, Emir UE, et al. Neurochemical and BOLD responses during neuronal activation measured in 
the human visual cortex at 7 Tesla. J Cereb Blood Flow Metab (2015) 35:60110. doi: $10.1038 / \mathrm{jcbfm} .2014 .233$

49. Chowdhury GM, Behar KL, Cho W, Thomas MA, Rothman DL, Sanacora G. (1) $\mathrm{H}-[(1)(3) \mathrm{C}]$-nuclear magnetic resonance spectroscopy measures of ketamine's effect on amino acid neurotransmitter metabolism. Biol Psychiatry (2012) 71:1022-5. doi: 10.1016/j.biopsych.2011.11.006

50. Wieronska JM, Pilc A. Metabotropic glutamate receptors in the tripartite synapse as a target for new psychotropic drugs. Neurochem Int (2009) 55:8597. doi: 10.1016/j.neuint.2009.02.019

51. Fuchs T, Jefferson SJ, Hooper A, Yee PH, Maguire J, Luscher B. Disinhibition of somatostatin-positive GABAergic interneurons results in an anxiolytic and antidepressant-like brain state. Mol Psychiatry (2017) 22:920-30. doi: 10.1038/ mp.2016.188

52. Li N, Lee B, Liu RJ, Banasr M, Dwyer JM, Iwata M, et al. mTOR-dependent synapse formation underlies the rapid antidepressant effects of NMDA antagonists. Science (2010) 329:959-64. doi: 10.1126/science.1190287

53. Popoli M, Yan Z, Mcewen BS, Sanacora G. The stressed synapse: the impact of stress and glucocorticoids on glutamate transmission. Nat Rev Neurosci (2011) 13:22-37. doi: $10.1038 / \mathrm{nrn} 3138$

54. Macqueen G, Frodl T. The hippocampus in major depression: evidence for the convergence of the bench and bedside in psychiatric research? Mol Psychiatry (2011) 16:252-64. doi: 10.1038/mp.2010.80

55. Bossù $\mathrm{P}$, Piras F, Palladino $\mathrm{I}$, Iorio $\mathrm{M}$, Salani $\mathrm{F}$, Ciaramella $\mathrm{A}$, et al. Hippocampal volume and depressive symptoms are linked to serum IL-18 in schizophrenia. Neurol - Neuroimmunol Neuroinflam (2015) 2:e111. doi: 10.1212/nxi.0000000000000111

56. Spalletta G, Piras F, Caltagirone C, Fagioli S. Hippocampal multimodal structural changes and subclinical depression in healthy individuals. J Affect Disord (2014) 152-154:105-12. doi: 10.1016/j.jad.2013.05.068

57. Frodl T, Jager M, Smajstrlova I, Born C, Bottlender R, Palladino T, et al. Effect of hippocampal and amygdala volumes on clinical outcomes in major depression: a 3-year prospective magnetic resonance imaging study. J Psychiatry Neurosci (2008) 33:423-30.

58. Abdallah CG, Salas R, Jackowski A, Baldwin P, Sato JR, Mathew SJ. Hippocampal volume and the rapid antidepressant effect of ketamine. J Psychopharmacol (2015a) 29:591-5. doi: 10.1177/0269881114544776
59. Ardalan M, Wegener G, Rafati AH, Nyengaard JR. S-Ketamine Rapidly Reverses Synaptic and Vascular Deficits of Hippocampus in Genetic Animal Model of Depression. Int J Neuropsychopharmacol (2017) 20:247-56. doi: 10.1093/ijnp/pyw098

60. Zanos P, Moaddel R, Morris PJ, Riggs LM, Highland JN, Georgiou P, et al. Ketamine and Ketamine Metabolite Pharmacology: Insights into Therapeutic Mechanisms. Pharmacol Rev (2018) 70:621-60. doi: 10.1124/pr.117.015198

61. Zanos P, Highland JN, Stewart BW, Georgiou P, Jenne CE, Lovett J, et al. $(2 \mathrm{R}, 6 \mathrm{R})$-hydroxynorketamine exerts mGlu2 receptor-dependent antidepressant actions. Proc Natl Acad Sci U S A (2019) 116:6441-50. doi: 10.1073/pnas.1819540116

62. Zarate CAJr., Niciu MJ. Ketamine for depression: evidence, challenges and promise. World Psychiatry (2015) 14:348-50. doi: 10.1002/wps.20269

63. Taylor MJ, Tiangga ER, Mhuircheartaigh RN, Cowen PJ. Lack of effect of ketamine on cortical glutamate and glutamine in healthy volunteers: a proton magnetic resonance spectroscopy study. J Psychopharmacol (2012) 26:733-7. doi: $10.1177 / 0269881111405359$

Conflict of Interest: RL received travel grants and/or conference speaker honoraria within the last 3 years from Bruker BioSpin MR, Heel, and support from Siemens Healthcare regarding clinical research using PET/MR. He is shareholder of BM Health $\mathrm{GmbH}$ since 2019. MS has received speaker honoraria from Janssen and Austroplant as well as travel grants and/or workshop participation from Janssen, Austroplant, AOP Orphan Pharmaceuticals, and Eli Lilly.

The remaining authors declare that the research was conducted in the absence of any commercial or financial relationships that could be construed as a potential conflict of interest.

Copyright (๑ 2020 Silberbauer, Spurny, Handschuh, Klöbl, Bednarik, Reiter, Ritter, Trost, Konadu, Windpassinger, Stimpfl, Bogner, Lanzenberger and Spies. This is an open-access article distributed under the terms of the Creative Commons Attribution License (CC BY). The use, distribution or reproduction in other forums is permitted, provided the original author(s) and the copyright owner(s) are credited and that the original publication in this journal is cited, in accordance with accepted academic practice. No use, distribution or reproduction is permitted which does not comply with these terms. 